\title{
Effect of polaronic charge transfer on band alignment at the $\mathrm{Cu} / \mathrm{TiO}_{2}$ interface
}

\author{
Keith P. McKenna* \\ Department of Physics, University of York, Heslington, York YO10 5DD, United Kingdom \\ (Received 28 June 2016; revised manuscript received 6 September 2016; published 27 October 2016)
}

\begin{abstract}
We present a first principles investigation into the electronic properties of an extended interface between rutile $\mathrm{TiO}_{2}$ and $\mathrm{Cu}$. We show that owing to the highly polarizable nature of $\mathrm{TiO}_{2}$, the interface is unstable to the spontaneous formation of small electron polarons at the interface. The resulting dipole leads to an increase in the conduction band offset by $0.4 \mathrm{eV}$ and the presence of a band of occupied states related to Ti $d$ states $1.4 \mathrm{eV}$ below the Fermi energy. This effect should be expected more generally at interfaces between highly polarizable oxides and metals but is missed by standard first principles approaches. Given the ubiquitous nature of such interfaces, this previously overlooked effect may have important implications for diverse applications across science and technology.
\end{abstract}

DOI: 10.1103/PhysRevB.94.155147

Small polarons (quasiparticles consisting of a localized charge carrier and an associated polarization field [1,2]) have been shown to form in a broad range of metal oxides. These include binary oxides such as $\mathrm{TiO}_{2}, \mathrm{HfO}_{2}, \mathrm{ZrO}_{2}$ $\mathrm{ZnO}, \mathrm{CeO}_{2}, \mathrm{Al}_{2} \mathrm{O}_{3}, \mathrm{Fe}_{2} \mathrm{O}_{3}$, as well as many manganites and titanates [3-14]. The formation and properties of polarons in these materials underpin diverse areas of physics including electron transport, photogenerated carrier dynamics, superconductivity, colossal magnetoresistance, and catalysis [15-20]. One usually considers the formation of small polarons resulting from the trapping of charge carriers introduced into a material either by intrinsic defects (such as vacancies or interstitials) [5,21,22], donor or acceptor impurities [23,24], or by photoexcitation $[12,25,26]$. The possibility of polaronic electron transfer at extended metal/metal-oxide interfaces is less commonly considered, but recent reports on highly reducible oxides such as $\mathrm{CeO}_{2}$ and $\mathrm{Fe}_{3} \mathrm{O}_{4}$ suggest it does occur [27-29]. Surprisingly, very little is known about the role of such polaronic charge transfer on interfacial band alignment, a key materials property that determines performance for diverse applications in electronics, sensing, and catalysis [15,16,30-32]. Indeed, while modern first principles theoretical calculations are routinely employed to model band offsets of such interfaces, the standard way of initializing calculations precludes the identification of polaronic charge transfer states even if they exist and are stable. Given the ubiquity of extended metal/metal-oxide interfaces across science and technology, understanding the extent of interfacial polaronic charge transfer and the consequences for the band alignment remain important but unexplored issues.

In this paper we investigate the electronic properties of an extended interface between $\mathrm{Cu}$ and $\mathrm{TiO}_{2}$ using first principles density functional theory (DFT). We show that this system is thermodynamically unstable to the polaronic transfer of electrons from $\mathrm{Cu}$ into $\mathrm{TiO}_{2}$, with electron polarons localizing

\footnotetext{
*keith.mckenna@york.ac.uk

Published by the American Physical Society under the terms of the Creative Commons Attribution 3.0 License. Further distribution of this work must maintain attribution to the author(s) and the published article's title, journal citation, and DOI.
}

on Ti sites close to the interface (forming $\mathrm{Ti}^{3+}$ species). The most stable structure has 2.54 transferred electrons per $\mathrm{nm}^{2}$, resulting in an increase in the conduction band offset (CBO) by $0.4 \mathrm{eV}$ with respect to an interface without polaronic charge transfer. These interfacial polarons are associated with electron states deep in the $\mathrm{TiO}_{2}$ gap $(1.4 \mathrm{eV}$ below the Fermi energy). While similar polaronic states can also be introduced by segregation of oxygen vacancies to the interface, they have a much weaker affect on the $\mathrm{CBO}$ since there is no net transfer of charge between the metal and the oxide. We stress that unless a concerted effort is made to identify such polaronic charge transfer interface configurations, they are missed by standard theoretical approaches. While the $\mathrm{Cu} / \mathrm{TiO}_{2}$ interface studied here provides a useful model system, the nature of the effect suggests similar behavior should be expected at interfaces involving other metal-oxide materials that can support small polarons.

The structural and electronic properties of extended metal/metal-oxide interfaces are often the subject of first principles investigations [30-37]. In general, such calculations are challenging since one must construct a commensurate supercell containing both the metal and metal oxide which usually requires imposing some degree of artificial strain. Correctly describing the polaronic transfer of charge at metal/metaloxide interfaces brings additional challenges associated with the need for correct treatment of the self-interaction (SI) error inherent to most DFT approaches. Electron trapping in $\mathrm{TiO}_{2}$ in particular has been extremely well studied both experimentally and theoretically $[6,10-12,24,38-43]$. These results suggest that a range of theoretical approaches (including DFT $+U$ and hybrid exchange-correlation functionals) are able to describe electron polarons correctly, providing any parameters controlling the degree of SI correction are chosen appropriately $[44,45]$.

The calculations presented in this paper are performed using spin polarized DFT, the projector augmented wave method, and the Perdew-Burke-Ernzerhof (PBE) exchange-correlation functional as implemented in the Vienna ab initio simulation package [46-48]. To correct for SI error on $\mathrm{Ti} 3 d$ states we employ the rotationally invariant DFT $+U$ method [49] with $U=4.2 \mathrm{eV}$, which was shown previously to give good agreement with the spectroscopic properties of oxygen vacancies at the (110) surface and employed to model electron 




FIG. 1. The $(3 \times 3) \mathrm{TiO}_{2}(001) \mid(4 \times 4) \mathrm{Cu}(001)$ supercell used to model the $\mathrm{TiO}_{2} \mid \mathrm{Cu}$ interface. Green, red, and gold spheres represent $\mathrm{Ti}, \mathrm{O}$, and $\mathrm{Cu}$ atoms, respectively. Atomic layers in $\mathrm{TiO}_{2}$ near the $\mathrm{Cu}$ interface are labeled L1-L3. A spin density isosurface associated with a polaron localized in layer 2 is shown along with the associated nearest-neighbor oxygen ion displacements. The figure produced using the VESTA software package [51].

polarons at $\mathrm{TiO}_{2}$ surfaces and a grain boundary $[38,40,41,50]$. The $4 s$ and $3 d$ electrons of $\mathrm{Ti}$ and $\mathrm{Cu}$, and the $2 s$ and $2 p$ electrons of $\mathrm{O}$ are treated as valence electrons and expanded in a plane wave basis. The bulk lattice constants of $\mathrm{Cu}$ $(a=3.64 \AA)$ and $\mathrm{TiO}_{2}(a=4.67 \AA$ and $c=3.03 \AA)$ are computed using a plane wave cutoff energy of $500 \mathrm{eV}$ and a well-converged Monkhorst-Pack grid $(11 \times 11 \times 11$ for $\mathrm{Cu}$ and $5 \times 5 \times 5$ for $\mathrm{TiO}_{2}$ ) and are in very good agreement with experiment (within 3\%). A planar interface between $\mathrm{TiO}_{2}(001)$ and $\mathrm{Cu}(001)$ is constructed by adjoining $3 \times 3$ unit cells of $\mathrm{TiO}_{2}(001)$ and $4 \times 4$ unit cells of $\mathrm{Cu}(001)$, allowing a commensurate interface to be formed with less than 3.5\% strain (applied to the $\mathrm{Cu}$ layer). Six atomic layers of both $\mathrm{Cu}$ and $\mathrm{TiO}_{2}$ are included (354 atoms in total) with a vacuum gap of $10 \AA$, as shown in Fig. 1 .

First, we calculate the structure and electronic properties of this interface in the standard way starting from a moreor-less homogeneous charge density (random wave functions) and self-consistently solving the Kohn-Sham equations. As expected, this results in a structure with no evidence of polaronic charge transfer into $\mathrm{TiO}_{2}$. There is of course still some local charge transfer near the interface resulting from equilibration of the Fermi energies in both materials [52]. Quantification of the amount of charge transferred depends on the method employed. For example, employing Bader analysis, we find approximately 1.2 electrons per unit cell ( 0.61 electrons per $\mathrm{nm}^{2}$ ) are transferred from $\mathrm{Cu}$ into $\mathrm{TiO}_{2}$ [53]. To explore the possibility of polaronic charge transfer, we also perform a series of calculations where oxygen ions in the initial structure are dilated by $0.08 \AA$ around prospective polaron sites, creating a precursor for electron localization. We also employ an occupation matrix control methodology in order to guide the self-consistent field minimization towards different polaronic charge transfer states [54]. In this way we obtain 27 different metastable configurations corresponding to the polaronic transfer of one electron to each Ti site within the first three layers of the interface (noting there are nine Ti ions in each layer). For all sites the polaronic charge transfer structures have a lower total energy by up to $0.30 \mathrm{eV}$. Figure 1 shows a spin density isosurface for the most stable configuration along

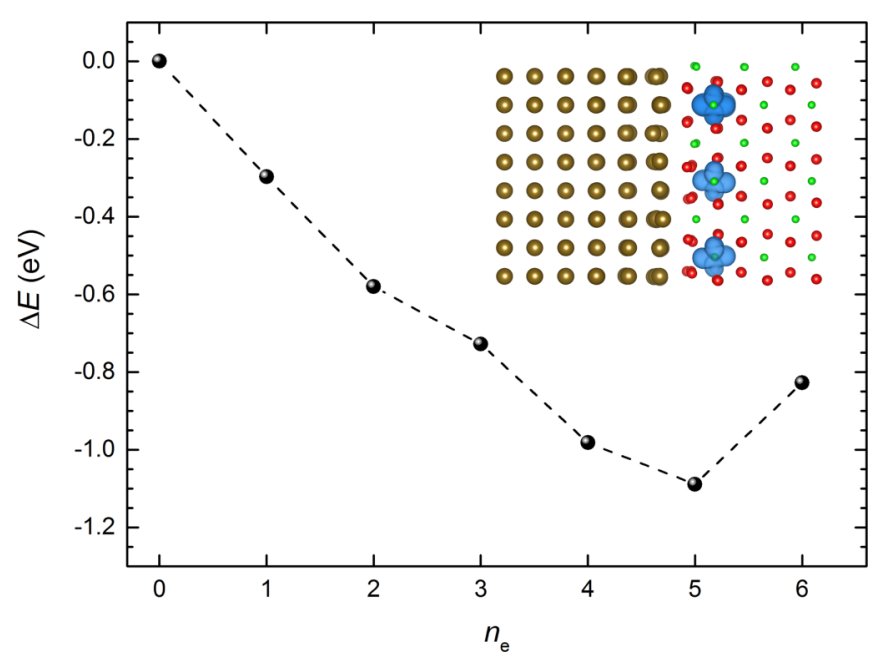

FIG. 2. Dependence of total energy on the number of electron polarons in $\mathrm{TiO}_{2}$ formed by charge transfer from $\mathrm{Cu}\left(n_{\mathrm{e}}\right) . \Delta E$ is the total energy relative to the energy of the ideal interface without electron polarons in $\mathrm{TiO}_{2}$. The inset shows the structure and electron spin density isosurfaces for the most stable $n_{\mathrm{e}}=5$ case.

with the characteristic polaronic distortion which involves an outward displacement of neighboring oxygen ions by $\sim 0.1 \AA$. We find that electron polarons in layer 2 are the most stable with energies $\Delta E_{2}=-0.24 \pm 0.08 \mathrm{eV}$ relative to the interface without polaronic charge transfer. The relative energies for polarons in layer 1 are $\Delta E_{1}=-0.16 \pm 0.01 \mathrm{eV}$ and in layer 3 are $\Delta E_{3}=-0.07 \pm 0.02 \mathrm{eV}$. The seven most stable polaron sites all reside within layer 2 . The high stability of polarons in layer 2 is natural since these sites benefit from a full coordination shell of oxygen ions needed for the polaronic distortion while also being close to the metal layer where the stabilizing effect of the image interaction will be more strongly felt.

We next explore the stability of the interface with respect to increasing the concentration of transferred electrons. Figure 2 shows how the total energy varies as the number of electron polarons in $\mathrm{TiO}_{2}\left(n_{\mathrm{e}}\right)$ is increased from 0 to 6 . The almost linear decrease of total energy for $n_{\mathrm{e}}<5$ is a reflection of relatively weak polaron-polaron interactions, consistent with the high dielectric constant of $\mathrm{TiO}_{2}$. For $n_{\mathrm{e}}>5$ the total energy begins to increase, suggesting polaron-polaron interactions are becoming significant at these densities $(>2.5$ polarons per $\mathrm{nm}^{2}$ ). We find the total energy is minimized for $n_{\mathrm{e}}=5$, which is more stable than the $n_{\mathrm{e}}=0$ case by $1.09 \mathrm{eV}$. Bader analysis indicates the total charge transferred into $\mathrm{TiO}_{2}$ is increased from $1.2\left(n_{\mathrm{e}}=0\right)$ to 3.2 electrons $\left(n_{\mathrm{e}}=5\right)$ per unit cell. Associated with the electron transfer and localization is also a magnetic moment of $4.9 \mu_{\mathrm{B}}\left(2.5 \mu_{\mathrm{B}} / \mathrm{nm}^{2}\right)$. We have explored alternative magnetic configurations, however, these were found either not to converge or to be less stable than the ferromagnetic solution.

We assess the effect of polaronic charge transfer on the electronic structure of the interface by calculating the density of states (DOS) for the $n_{\mathrm{e}}=0$ and $n_{\mathrm{e}}=5$ structures obtained above, shown in Fig. 3. First, we note there is little effect of charge transfer on the $\mathrm{Cu}$ DOS, indicating that the $\mathrm{Cu}$ 


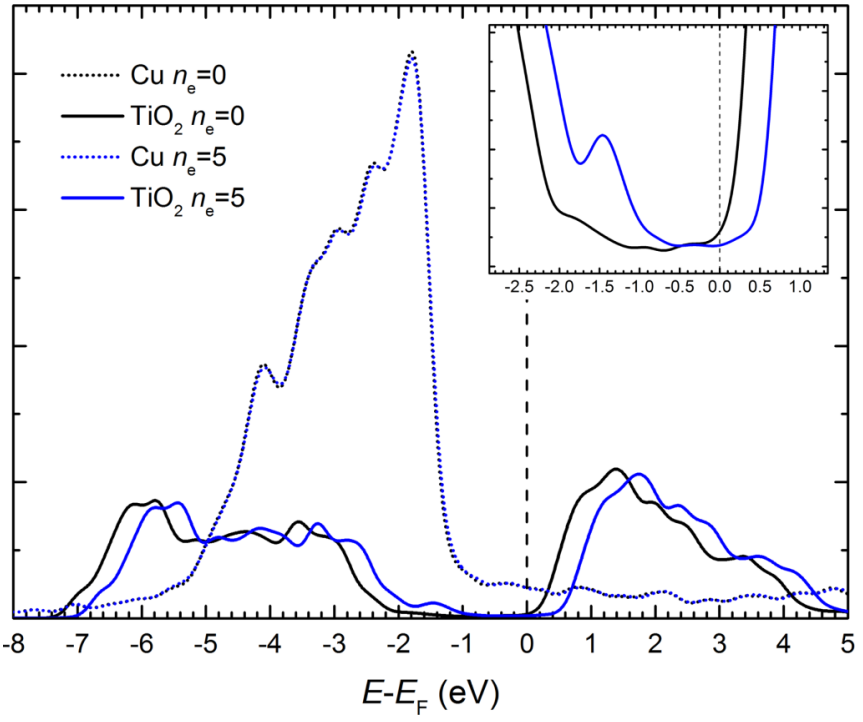

FIG. 3. Electronic density of states (spin up) projected onto $\mathrm{Cu}$ and $\mathrm{TiO}_{2}$ regions for the $n_{\mathrm{e}}=0$ and $n_{\mathrm{e}}=5$ structures of the $\mathrm{TiO}_{2}(001) \mid \mathrm{Cu}(001)$ interface. The inset shows a zoomed-in view of the spin-up electronic states highlighting the occupied polaron states around $1.4 \mathrm{eV}$ below the Fermi energy. The density of states for both spin eigenstates is included in the Supplemental Material (Fig. S1) [55].

layer represents such a sufficiently large electron reservoir that the position of the Fermi energy is not significantly affected by the removal of electrons. However, the $\mathrm{TiO}_{2}$ conduction band offset is seen to clearly increase from 0.15 to $0.55 \mathrm{eV}$ following electron transfer. For the $n_{\mathrm{e}}=0$ case the presence of metal induced gap states responsible for the equilibration of Fermi energies can be seen in the inset of Fig. 3. For $n_{\mathrm{e}}=5$ there is an additional band of states at about $1.4 \mathrm{eV}$ below the Fermi energy which is associated with the interfacial polarons. It is the additional dipole associated with these filled states that gives rise to the increase in CBO. One can also introduce $\mathrm{Ti}^{3+}$ species at the interface by the introduction of oxygen vacancies. For example, a single vacancy introduced near the interface creates two electron polarons. As the concentration of vacancies in increased further, additional polarons are introduced. For six oxygen vacancies near the interface, five localized electron polarons are created. The remaining seven electrons are found to be delocalized over $\mathrm{Cu}$ and $\mathrm{Ti}$ ions at the interface. However, the CBO in this situation is barely changed from the $n_{\mathrm{e}}=0$ case, confirming the key role played by the polaronic charge transfer and associated dipole (see Fig. S2, Supplemental Material [55]). The electronic properties of these interfaces have also been calculated using the Heyd, Scuseria, and Ernzerhof (HSE) hybrid functional which includes nonlocal exchange in order to correct for the SI error (without geometry optimization due to the high computational expense of these calculations) [56]. The results are qualitatively unchanged, i.e., an increase in CBO from 0.10 to $0.45 \mathrm{eV}$, but with an increased band gap closer to the experimental value (see Fig. S3, Supplemental Material [55]).

In order to summarize these findings, we present a schematic in Fig. 4 to illustrate the effect of polaronic charge (a)
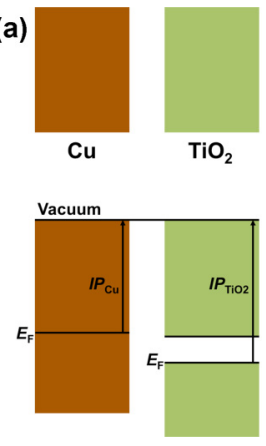

(b)
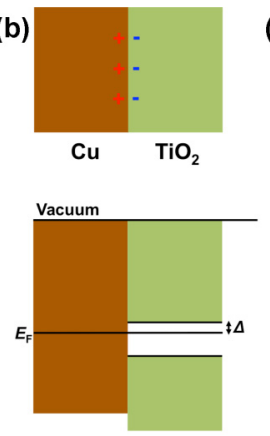

(c)
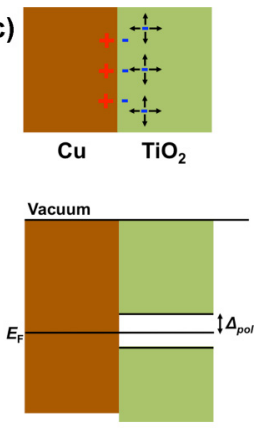

FIG. 4. Schematic illustrating the effect of polaronic charge transfer on the conduction band offset of a $\mathrm{Cu} / \mathrm{TiO}_{2}$ interface. (a) The ionization potential (IP) of an isolated slab of $\mathrm{TiO}_{2}$ is significantly higher than that of $\mathrm{Cu}$. (b) When the two slabs are brought together to form an interface, the Fermi level in the two materials becomes equilibrated as a result of structural relaxation at the interface and partial charge transfer from $\mathrm{Cu}$ to $\mathrm{TiO}_{2}$, resulting in a conduction band offset $\Delta$. (c) If one considers the more stable electronic configuration involving polaronic electron transfer from $\mathrm{Cu}$ creating $\mathrm{Ti}^{3+}$ species at the interface, the additional dipole results in an increase in the conduction band offset $\Delta_{\mathrm{pol}}$.

transfer on the $\mathrm{CBO}$ at the $\mathrm{Cu} / \mathrm{TiO}_{2}$ interface. If we first consider the $\mathrm{Cu}$ and $\mathrm{TiO}_{2}$ slabs in isolation [Fig. 4(a)], there is a difference in the ionization potential (IP) of the two materials. Performing a calculation of the IP for the isolated slabs from Fig. 1, we obtain $\mathrm{IP}_{\mathrm{Cu}}=4.7 \mathrm{eV}$ and $\mathrm{IP}_{\mathrm{TiO}_{2}}=6.1 \mathrm{eV}$ (using the PBE exchange correlation functional), a difference of $1.4 \mathrm{eV}$. The value for $\mathrm{Cu}$ is in excellent agreement with experiment [57]. Experimental determination of the work function for ideal undoped rutile $\mathrm{TiO}_{2}$ is less straightforward with reported values ranging from 7 to $8 \mathrm{eV}$ [58,59]. When they are brought into contact [Fig. 4(b)], there is a modification of the local structure near the interface and a self-consistent redistribution of charge such that the Fermi energies in the two materials are equilibrated. If one does not consider the possibility of polaronic electron transfer, the resulting $\mathrm{CBO}(\Delta)$ is $0.15 \mathrm{eV}$. However, the more stable polaronic configuration corresponding to the transfer of five electrons into layer 2 has a $\mathrm{CBO}\left(\Delta_{\text {pol }}\right)$ of $0.55 \mathrm{eV}$, an increase of $0.4 \mathrm{eV}$.

At this point we would like to discuss some of the factors which may affect the accuracy of the results presented above. One of the most challenging issues is the elimination of SI error, which is important for an accurate description of electron trapping energies. Here, we have employed the DFT $+U$ approach with a parametrization that has been applied in a number of previous studies of electron trapping in $\mathrm{TiO}_{2}$ and tested in comparison to experiment $[38,40,41,50]$. While this approach does lead to a slight underestimation of the $\mathrm{TiO}_{2}$ band gap, our comparison with the more computationally demanding nonlocal HSE exchange-correlation functional suggests this does not adversely affect the calculated CBO. Importantly, the PBE functional employed in the DFT $+U$ approach ensures a very accurate description of the structure and electronic properties of $\mathrm{Cu}$, a weakness with many nonlocal functionals. While different exchange-correlation functionals and approaches may predict slightly different CBOs, the qualitative result should be unaffected, namely, 
that an interface between a low work functional metal and a highly polarizable metal oxide will likely lead to the formation of interfacial electron polarons and an increased CBO.

Direct experimental evidence for the formation of polarons at metal/metal-oxide interfaces is scarce and in general it is difficult to distinguish polarons arising from charge transfer from those due to oxygen vacancies. A recent work employing electron energy loss spectroscopy detected the presence of $\mathrm{Ce}^{3+}$ at the $\mathrm{Pt} / \mathrm{CeO}_{2}$ interface which was attributed to electron transfer from the metal [29]. Earlier work also suggested $\mathrm{Pt}$ nanoparticles could induce the formation of $\mathrm{Ce}^{3+}$ when deposited on a $\mathrm{CeO}_{2}$ support [27]. Conversely, the cationic charging of $\mathrm{Au}$ atoms on reduced $\mathrm{TiO}_{2}$ thin films has been observed [60]. We are not aware of similar experimental observations for $\mathrm{Cu} / \mathrm{TiO}_{2}$ interfaces, but our first principles calculations suggest that interfacial polarons are likely to be present. In principle, the unpaired spin associated with interfacial polarons could be detected directly by electron spin resonance spectroscopy. It has been demonstrated that this method can be applied to thin metal supported oxide films to characterize relatively low concentrations of paramagnetic species [61,62]. Another signature of the interfacial polarons is the band of deep occupied states predicted at around $1.4 \mathrm{eV}$ below the Fermi energy which could be detected spectroscopically.

In summary, using first principles theoretical calculations, we have shown that the $\mathrm{TiO}_{2}(001) / \mathrm{Cu}(001)$ interface is unstable to the spontaneous formation of significant concentrations of small electron polarons $\left(\mathrm{Ti}^{3+}\right)$ due to electron transfer from $\mathrm{Cu}$. The resulting dipole at the interface increases the conduction band offset by $0.4 \mathrm{eV}$. This effect would be missed using standard first principles approaches and suggests extra care must be taken when modeling such interfaces. In particular, it may be necessary to initialize the interface calculation with a locally distorted structure in order to obtain the most stable electronic configuration, e.g., as shown in Ref. [29] for the $\mathrm{Pt} / \mathrm{CeO}_{2}$ interface. One should expect a similar effect whenever a low work function metal is brought into contact with a highly polarizable metal oxide capable of supporting small polarons (e.g., $\mathrm{TiO}_{2}, \mathrm{HfO}_{2}, \mathrm{ZrO}_{2}$, and $\mathrm{SrTiO}_{3}$ ). This places an intrinsic restriction of the conduction band offset one can achieve between metals and highly polarizable oxides with important practical consequences for the design of a range of technological devices, e.g., metal contacts in dynamic random access memory devices and supercapacitors for energy storage. Electron polarons induced by charge transfer from the metal are bound to the interface by at least $0.1 \mathrm{eV}$, but at elevated temperatures or under bias could diffuse and play a role in transport or electrochemical processes. The interfacial polaron formation we describe adds to the rich physics of polarons in metal oxides with both fundamental and practical significance.

We acknowledge support from EPSRC (EP/K003151) and COST Action CM1104 "Reducible oxide chemistry, structure and functions" supported by COST (European Cooperation in Science and Technology). This work made use of the facilities of Archer, the UK's national high-performance computing service, via our membership in the UK HPC Materials Chemistry Consortium, which is funded by EPSRC (EP/L000202). All data created during this research are available by request from the University of York Research database http://dx.doi.org/10.15124/40d9456a-98214c81-b336-00287058b2e5
[1] T. Holstein, Ann. Phys. 8, 343 (1959).

[2] A. M. Stoneham, IEEE Trans. Dielectr. Electr. Insul. 4, 604 (1997).

[3] S. Yoon, H. L. Liu, G. Schollerer, S. L. Cooper, P. D. Han, D. A. Payne, S.-W. Cheong, and Z. Fisk, Phys. Rev. B 58, 2795 (1998).

[4] C. P. Adams, J. W. Lynn, Y. M. Mukovskii, A. A. Arsenov, and D. A. Shulyatev, Phys. Rev. Lett. 85, 3954 (2000).

[5] O. F. Schirmer, J. Phys.: Condens. Matter 18, R667 (2006).

[6] N. A. Deskins and M. Dupuis, Phys. Rev. B 75, 195212 (2007).

[7] S. Wright and R. C. Barklie, J. Appl. Phys. 106, 103917 (2009).

[8] K. P. McKenna, M. J. Wolf, A. L. Shluger, S. Lany, and A. Zunger, Phys. Rev. Lett. 108, 116403 (2012).

[9] M. J. Wolf, K. P. McKenna, and A. L. Shluger, J. Phys. Chem. C 116, 25888 (2012).

[10] S. Yang, A. T. Brant, N. C. Giles, and L. E. Halliburton, Phys. Rev. B 87, 125201 (2013).

[11] M. Setvin, C. Franchini, X. Hao, M. Schmid, A. Janotti, M. Kaltak, C. G. Van de Walle, G. Kresse, and U. Diebold, Phys. Rev. Lett. 113, 086402 (2014).

[12] H. Sezen, M. Buchholz, A. Nefedov, C. Natzeck, S. Heissler, C. Di Valentin, and C. Wöll, Sci. Rep. 4, 3808 (2014).

[13] A. Janotti, J. B. Varley, M. Choi, and C. G. Van de Walle, Phys. Rev. B 90, 085202 (2014).
[14] Z. Wang, S. McKeown Walker, A. Tamai, Y. Wang, Z. Ristic, F. Y. Bruno, A. de la Torre, S. Ricco, N. C. Plumb, M. Shi et al., Nat. Mater. 15, 835 (2016).

[15] K. Schierbaum, U. Kirner, J. Geiger, and W. Göpel, Sens. Actuators, B 4, 87 (1991).

[16] M. D. Pickett, D. B. Strukov, J. L. Borghetti, J. J. Yang, G. S. Snider, D. R. Stewart, and R. S. Williams, J. Appl. Phys. 106, 074508 (2009).

[17] R. Waser and M. Aono, Nat. Mater. 6, 833 (2007).

[18] B. O'Regan and M. Grätzel, Nature (London) 353, 737 (1991).

[19] L. M. Peter, J. Phys. Chem. Lett. 2, 1861 (2011).

[20] A. Fujishima and K. Honda, Nature (London) 238, 37 (1972).

[21] P. Deák, B. Aradi, and T. Frauenheim, Phys. Rev. B 92, 045204 (2015).

[22] K. P. McKenna and D. M. Ramo, Phys. Rev. B 92, 205124 (2015).

[23] S. Lany and A. Zunger, Phys. Rev. B 80, 085202 (2009).

[24] B. J. Morgan, D. O. Scanlon, and G. W. Watson, J. Mater. Chem. 19, 5175 (2009).

[25] A. J. E. Rettie, W. D. Chemelewski, D. Emin, and C. B. Mullins, J. Phys. Chem. Lett. 7, 471 (2016).

[26] C. Di Valentin and A. Selloni, J. Phys. Chem. Lett. 2, 2223 (2011). 
[27] G. N. Vayssilov, Y. Lykhach, A. Migani, T. Staudt, G. P. Petrova, N. Tsud, T. Skála, A. Bruix, F. Illas, K. C. Prince et al., Nat. Mater. 10, 310 (2011).

[28] Z. Novotný, G. Argentero, Z. Wang, M. Schmid, U. Diebold, and G. S. Parkinson, Phys. Rev. Lett. 108, 216103 (2012).

[29] P. Luches, L. Giordano, V. Grillo, G. C. Gazzadi, S. Prada, M. Campanini, G. Bertoni, C. Magen, F. Pagliuca, G. Pacchioni et al., Adv. Mater. Interfaces 2, 1500375 (2015).

[30] W. H. Butler, X.-G. Zhang, T. C. Schulthess, and J. M. MacLaren, Phys. Rev. B 63, 054416 (2001).

[31] L. Giordano, F. Cinquini, and G. Pacchioni, Phys. Rev. B 73, 045414 (2006).

[32] J. Robertson, Phys. Status Solidi 207, 261 (2010).

[33] J. Goniakowski and C. Noguera, Interface Sci. 12, 93 (2004).

[34] A. A. A. Demkov, Phys. Rev. B 74, 085310 (2006).

[35] T. Tamura, S. Ishibashi, K. Terakura, and H. Weng, Phys. Rev. B 80, 195302 (2009).

[36] S. R. Bradley, K. P. McKenna, and A. L. Shluger, Microelectron. Eng. 109, 346 (2013).

[37] S. Ling, M. B. Watkins, and A. L. Shluger, J. Phys. Chem. C 117, 5075 (2013).

[38] B. J. Morgan and G. W. Watson, J. Phys. Chem. C 113, 7322 (2009).

[39] P. Deák, B. Aradi, and T. Frauenheim, Phys. Rev. B 86, 195206 (2012).

[40] S. K. Wallace and K. P. McKenna, Adv. Mater. Interfaces 1, 1400078 (2014).

[41] S. K. Wallace and K. P. McKenna, J. Phys. Chem. C 119, 1913 (2015).

[42] N. A. Deskins, R. Rousseau, and M. Dupuis, J. Phys. Chem. C 113, 14583 (2009).

[43] N. A. Deskins, R. Rousseau, and M. Dupuis, J. Phys. Chem. C 115, 7562 (2011).

[44] M. Cococcioni and S. de Gironcoli, Phys. Rev. B 71, 035105 (2005).
[45] S. Lany and A. Zunger, Phys. Rev. B 81, 205209 (2010).

[46] G. Kresse and J. Furthmüller, Phys. Rev. B 54, 11169 (1996).

[47] G. Kresse and J. Furthmüller, Comput. Mater. Sci. 6, 15 (1996).

[48] J. P. Perdew, K. Burke, and M. Ernzerhof, Phys. Rev. Lett. 77, 3865 (1996).

[49] S. L. Dudarev, G. A. Botton, S. Y. Savrasov, C. J. Humphreys, and A. P. Sutton, Phys. Rev. B 57, 1505 (1998).

[50] B. J. Morgan and G. W. Watson, Surf. Sci. 601, 5034 (2007).

[51] K. Momma and F. Izumi, J. Appl. Crystallogr. 44, 1272 (2011).

[52] J. Tersoff, Phys. Rev. Lett. 52, 465 (1984).

[53] G. Henkelman, A. Arnaldsson, and H. Jónsson, Comput. Mater. Sci. 36, 354 (2006).

[54] J. P. Allen and G. W. Watson, Phys. Chem. Chem. Phys. 16, 21016 (2014).

[55] See Supplemental Material at http://link.aps.org/supplemental/ 10.1103/PhysRevB.94.155147 for spin-resolved electronic densities of states calculated at the DFT $+U$ and HSE levels of theory.

[56] J. Heyd, G. E. Scuseria, and M. Ernzerhof, J. Chem. Phys. 118 8207 (2003).

[57] P. O. Gartland, S. Berge, and B. J. Slagsvold, Phys. Rev. Lett. 28, 738 (1972).

[58] L. Kavan, M. Grätzel, S. E. Gilbert, C. Klemenz, and H. J. Scheel, J. Am. Chem. Soc. 118, 6716 (1996).

[59] D. O. Scanlon, C. W. Dunnill, J. Buckeridge, S. A. Shevlin, A. J. Logsdail, S. M. Woodley, C. R. A. Catlow, M. J. Powell, R. G. Palgrave, I. P. Parkin et al., Nat. Mater. 12, 798 (2013).

[60] A. S. Wörz, U. Heiz, F. Cinquini, and G. Pacchioni, J. Phys. Chem. B 109, 18418 (2005).

[61] M. Sterrer, E. Fischbach, T. Risse, and H.-J. Freund, Phys. Rev. Lett. 94, 186101 (2005).

[62] M. Yulikov, M. Sterrer, T. Risse, and H.-J. Freund, Surf. Sci. 603, 1622 (2009). 